\title{
FUTURE EXPERIMENTS WITH NEUTRINO SUPERBEAMS, BETA-BEAMS, AND NEUTRINO FACTORIES
}

\author{
D. A. HARRIS \\ Fermi National Accelerator Laboratory P.O. Box 500 Batavia, Illinois, USA \\ E-mail:dharris@fnal.gov
}

\begin{abstract}
This report describes the goals of the next generations of accelerator-based neutrino experiments, and the various strategies that are being considered to achieve those goals. Because these next steps in the field are significantly different from the current or previous steps, novel techniques must be considered for both the detectors and the neutrino beams themselves. We consider not only conventional neutrino beams created by decays of pions, but also those which could be made by decays of beams of relativistic isotopes (so-called "beta-beams") and also by decays of beams of muons (neutrino factories).
\end{abstract}

\section{Introduction}

The current generation of neutrino experiments (K2K, MINOS, MiniBooNE, OPERA, and ICARUS) is focusing on verifying the oscillation framework and making the first precision measurements of the neutrino mass splittings. Specifically, they are each optimized to search for a particular signal which is predicted to be several statistical standard deviations away from zero, and which is predicted based on previous experimental evidence. The next step in this field, however, is to search for the last unmeasured element in the leptonic mixing matrix, and here there is essentially no theoretical guidance for how big a signal to expect.

Assuming that there are only three generations of neutrinos contributing to oscillations, the leptonic mixing matrix $U$, which converts from the flavor to the mass eigenstates, can be parameterized with three angles $\theta_{12}, \theta_{23}$, and $\theta_{13}$ and a CP-violating phase $\delta$.

$$
U=\left(\begin{array}{ccc}
C_{12} C_{13} & S_{12} C_{13} & S_{13} e^{-i \delta} \\
-S_{12} C_{23}+\ldots & C_{12} C_{23}+\ldots & S_{23} C_{13} \\
S_{12} S_{23}+\ldots & -C_{12} S_{23}+. . & C_{23} C_{13}
\end{array}\right)
$$

where $S_{i j}=\sin \theta_{i j}$ and $C_{i j}=\cos \theta_{i j}$. The first two mixing angles $\left(\theta_{12}, \theta_{23}\right)$ are known to be large from the solar and atmospheric disappearance measurements, respectively. The third mixing angle, $\theta_{13}$ can be measured by either looking for electron disappearance (for example, at a reactor experiment at a few kilometers distance), or by measuring $\nu_{\mu} \rightarrow \nu_{e}$ at the "atmospheric mass splitting" $\left(\Delta m_{23}^{2}\right)$. The current most sensitive limit for $\sin ^{2} 2 \theta_{13}$ is roughly 0.1 , and comes from the $\mathrm{CHOOZ}$ reactor experiment 1 .
In the limit where the solar mass splitting $\left(\Delta m_{12}^{2}\right)$ is zero, the probability for muon neutrinos to oscillate to electron neutrinos in vacuum is

$$
P\left(\nu_{\mu} \rightarrow \nu_{e}\right)=\sin ^{2} \theta_{23} \sin ^{2} 2 \theta_{13} \sin ^{2}\left(\frac{\Delta m_{23}^{2} L}{4 E}\right)
$$

where $L$ and $E$ are the distance the neutrino has travelled and its energy, respectively.

So by looking for electron appearance in a muon neutrino beam (or muon appearance in an electron neutrino beam), one can get an indication for $\sin ^{2} 2 \theta$ to be non-zero. However, once a signal for $\nu_{\mu} \leftrightarrow \nu_{e}$ is seen, there is much more to be measured in this sector. At that point all the additional terms not given in equation 2 become important. By looking at the oscillation probability for both neutrinos and antineutrinos one can determine the neutrino mass hierarchy, and also start to search for CP violation.

For neutrinos propagating through the vacuum, the asymmetry in the neutrino and antineutrino probabilities can be expressed as follows:

$$
\begin{aligned}
A(\mu e) & =\frac{P\left(\nu_{\mu} \rightarrow \nu_{e}\right)-P\left(\bar{\nu}_{\mu} \rightarrow \bar{\nu}_{e}\right)}{P\left(\nu_{\mu} \rightarrow \nu_{e}\right)+P\left(\bar{\nu}_{\mu} \rightarrow \bar{\nu}_{e}\right)} \\
& =\frac{\Delta m_{12}^{2} L}{E} \frac{\sin \delta}{\sin \theta_{13}}<1
\end{aligned}
$$

where $L$ and $E$ are the experiment baseline and neutrino energy, respectively, $\Delta m_{12}^{2}$ is the mass squared difference that has been seen in the solar neutrino sector, and $\delta$ is the CP-violating phase in the leptonic mixing matrix. Note that although that the asymmetry must by definition be less than one, as $\theta_{13}$ gets smaller the asymmetry actually gets larger. Also, if an experiment is planned to run with longer baseline, say the second oscillation maximum $\left(\Delta m_{23}^{2} L / 4 E=\right.$ $3 \pi / 2)$ instead of the first $\left(\Delta m_{23}^{2} L / 4 E=\pi / 2\right)$, then 
the $\mathrm{CP}$ violating effects are three times as large as at the first ${ }^{2}$.

When electron neutrinos and antineutrinos travel through the earth, however, they scatter off electrons in the earth, which itself causes an asymmetry, even without any CP-violating phase in the mixing matrix. In the limit of $\Delta m_{12}^{2}=0$ (which would also imply no CP violation), the asymmetry for an experiment running near the oscillation maximum (i.e. where $\Delta m_{23}^{2} L / 4 E$ is near $\pi / 2$ ) is ${ }^{3}$ :

$$
\begin{aligned}
A(\mu e) & =\frac{2 E}{E_{R}}\left(1-\left[\frac{\pi}{2}\right]^{2} \frac{E-E_{o m}}{E}\right) \\
E_{R} & =\frac{\Delta m_{23}^{2}}{2 \sqrt{2} G_{F} \rho_{e}} \approx 11 G e V \\
E_{o m} & =\frac{\Delta m^{2} L}{2 \pi}
\end{aligned}
$$

In the experiments to be described in this document, the matter effects alone will cause this asymmetry to be anywhere from a few per cent to close to unity. By measuring the sign of these matter effects, one can determine whether or not $\Delta m_{23}^{2}$ is positive or negative, which will determine whether or not neutrinos follow the same mass pattern as the charged fermions. Most theories predict that the mass hierarchy is "normal" (or $\Delta m_{23}^{2}$ is positive), but that fact makes this hierarchy even more important to determine experimentally.

There is also information in the precise value of the atmospheric mixing angle, otherwise known as $\theta_{23}$, which is currently within experimental errors of $\pi / 4$. How much this angle differs from $\pi / 4$ is important for two reasons. The fact that it is so near to $\pi / 4$ in the first place implies some underlying symmetry, whose origin we do not yet know, and yet how different $\theta_{23}$ is from $\pi / 4$ implies how strongly that symmetry could be broken. Imagine how different the field of particle physics would be today if we did not know how far the long-lived neutral kaon was from not being simply an exactly equal admixture of $K^{0}$ and $\bar{K}_{0}$ ! Also, from a purely experimental point of view, the farther $\theta_{23}$ is from $\pi / 4$ the harder it becomes to extract the other matrix elements from measurements of probabilities. This is because while the $\nu_{\mu}$ disappearance probability is a function of $\sin ^{2} 2 \theta_{23}$, the appearance probabilities are functions only of $\sin ^{2} \theta_{23}$, which by definition is more poorly constrained than $\theta_{23}$, for values near $\pi / 4$. So, although the primary motivation of the experiments described in this report is a search for $\theta_{13}$ and not an improved measurement of $\sin ^{2} 2 \theta_{23}$, one would want and expect a new level of precision here as well.

Before describing each of the experiments individually, however, this report will discuss general techniques for making neutrino beams in the first place, and then describe a few detectors which are being considered in either the near or far term future.

\section{Techniques for Making Neutrino Beams}

There are currently three vastly different strategies for making neutrino beams, and can be classified by what particle is decaying to produce the neutrinos. Conventional beams, made from pion decays, have been used in particle physics for decades. However, this technique inherently makes a beam which contains both muon and electron neutrinos, which ultimately limits how small a $\nu_{\mu} \rightarrow \nu_{e}$ oscillation probability can be measured. Beta Beams, made from decays of beams of radioactive isotopes, are purely $\nu_{e}$ or $\bar{\nu}_{e}$, depending on what isotope is decaying ${ }^{4}$. Finally, neutrino factory beams come from positively or negatively charged muon decays, and contain roughly equal amounts of $\nu_{\mu}$ and $\bar{\nu}_{e}$, or of $\bar{\nu}_{\mu}$ and $\nu_{e}$, depending on the charge of the muons ${ }^{5}$. Although these last beams seem to be the most mixed, in fact they are the easiest to use from the detector standpoint: by measuring the presence of a muon in the far detector one has measured the flavor of the final state neutrino, and by measuring that muon's charge one has determined the flavor of the initial state neutrino in the beam, since the electron-flavor neutrinos always have the opposite helicity as the muon-flavor neutrinos.

\subsection{Conventional Neutrino Beams}

Conventional neutrino beams are created by bombarding a target with as many protons as can be provided, and then focusing the produced mesons (mostly pions and some kaons) into an evacuated decay region where they are allowed to decay. The mesons will decay primarily to muons and muon neutrinos, and because the decay is a two-body decay the energy of the neutrino is directly related to the parent meson energy and the angle between the parent 
meson direction and the neutrino. In order to produce a beam of antineutrinos, the polarity of the focusing devices must be reversed, and one arrives at a $\bar{\nu}_{\mu}$ beam. In either case there is always some contamination of $\nu_{e}$ or $\bar{\nu}_{e}$, since both the kaons and daughter muons can undergo three-body decays which produce electron neutrinos.

In the near term future, two experiments are being proposed which use detectors which are not located on the beamline axis. Because of the two-body nature of the pion decay, there is an angle with respect to the focused pion direction where a broad band of pion energies will produce a narrow band of neutrino energies. This is in contrast to an "on axis" neutrino experiment, where the neutrino energy at the far detector is directly proportional to the pion energy in the beamline. So although the total event rates at an off axis location are considerably reduced, the $\nu_{\mu}$ event rate in a narrow energy band is in fact much larger than in the same energy band for an on axis detector. Because the electron neutrinos are produced in three-body decays, this event peaking is not nearly as strong, and as a result not only the signal in a narrow energy band improves, but the signal to background ratio is much better than in the on axis case.

For the farther term future, "on axis" beams are again being considered, where the primary motivation is not to extend the search for ever smaller values of $\theta_{13}$, but to measure, once $\nu_{\mu} \rightarrow \nu_{e}$ is found, the neutrino mass hierarchy through matter effects and to begin the search for $\mathrm{CP}$ violation in the lepton sector.

\subsection{Beta-beams}

Beta-beams are a relatively new idea for making extremely pure electron neutrino or antineutrino beams using accelerated beams of radioactive ions ${ }^{4}$. For example, if one were to produce and accelerate an intense beam of ${ }^{6} \mathrm{He} \quad\left({ }^{18} \mathrm{Ne}\right)$ ions to $139(55) \mathrm{GeV} / \mathrm{u}$, then one would have a wide-band energy neutrino (antineutrino) beam with an energy up to about $1 \mathrm{GeV}$. The average neutrino beam energies are on the order of a few hundred $\mathrm{MeV}$, and therefore a far detector would have to be located about one hundred $\mathrm{km}$ away to adress the atmospheric oscillation frequency. This proposal is being studied in depth at CERN, where the ISOL system would be used to pro- duce roughly $10^{17}$ ions per year. These ions would then be accelerated in two cyclotrons, the Proton Synchrotron (PS), and finally the Super Proton Synchrotron (SPS), after which they would be injected into a decay ring. To maximize the number of ions decaying while pointing towards a far detector, the ring could consist of two straight sections that are 2,500 m long, connected by short arcs ${ }^{6}$.

Because the beta-beam neutrino energies and event rates at a far detector are comparable with those of atmospheric neutrinos, the relativistic ions must be bunched once they arrive at the decay ring. Although this complicates the acceleration, it allows for twice as many measurements to be made. By storing both the ${ }^{6} \mathrm{He}$ and ${ }^{18} \mathrm{Ne}$ ions in the ring at the same time, (in which case they would have to be at the same momentum), one could produce bunched beams of either neutrinos or antineutrinos. By using relative timing between the beamline and the far detector events one could then measure neutrino and antineutrino oscillation probabilities in the same run ${ }^{7}$.

\subsection{Neutrino Factories}

Finally, the term "neutrino factory" has been used to describe neutrino beams created by the decays of high energy muons which are again stored in elongated rings. A neutrino factory would produce beams of equal numbers of $\nu_{\mu}$ and $\bar{\nu}_{e}\left(\bar{\nu}_{\mu}\right.$ and $\left.\nu_{e}\right)$ when negative (positive) muons are stored in the ring. With positively charged muons circulating in the ring, one could measure $\nu_{e} \rightarrow \nu_{\mu}$ simply by looking for a neutrino interaction in the far detector with a negatively charged muon emerging. The $\bar{\nu}_{\mu}$ neutrinos which are also in this same beam would produce only positively charged muons. Although neutrino interactions frequently produce pions of both charges, these pions can very easily be distinguished from muons once the muons are above a few $\mathrm{GeV}$. Neutrino factories are therefore being considered with muon energies from $20 \mathrm{GeV}$ up to $50 \mathrm{GeV}$, which produce wide band neutrino energies with an average of about a third of the muon energy. For most studies, the number of muons decaying in the ring per year is on the order of $10^{20}$. The baselines considered for these beams range from about $700 \mathrm{~km}$ to $8000 \mathrm{~km}$.

A neutrino factory begins with a high intensity 
beam of protons (like the one needed for a neutrino superbeam, for example) which is sent to a target which has been optimized to make low energy pions. The produced pions are then collected through solenoid focusing while they decay, at which point they are a dense cloud of muons (with a broad range of energies). By phase rotation the muon energy spread is reduced, at which point the muons can be accelerated. Because of the short muon lifetime the muons must be accelerated very quickly, and herein lies the biggest technological challenge in building a neutrino factory.

However, the excitement that has been generated in this field over the possibility of building a neutrino factory is due to the fact that for a given proton power, a $30 \mathrm{GeV}$ neutrino factory would produce about a factor of three more neutrinos than a superbeam at a comparable energy ${ }^{8}$. Also, the oscillation searches done with that beam would have more than an order of magnitude lower backgrounds, simply by using a detector that could distinguish the charge of an outgoing muon.

\subsection{Beamline Summary}

Figure 1 shows the neutrino fluxes per Megawatt (with the exception of the $\beta$-beam) for several of the proposals to be described in this report. Because the fluxes that are relevant are those at the location of the experiment, the fluxes given are for each of the different baselines being considered.

\section{Techniques for Detecting Neutrinos}

Clearly for an oscillation experiment, a detector must be able to identify both the flavor and the energy of the incoming neutrino. The separation between an electron and a muon is trivial in most detectors, but the challenge is in both reconstructing the neutrino energy, and separating muons or electrons from other final state particles which are often produced in neutrino interactions (i.e. charged or neutral pions).

Before listing the detectors being considered for the next generation of neutrino experiments, it is useful to list the different interactions that acceleratorproduced neutrinos (i.e. at or above a few hundred $\mathrm{MeV}$ in energy) can have with matter. First of all, there is the quasielastic interaction, which dominates the total cross section below about $700 \mathrm{MeV}$ : this oc- curs when a neutrino simply exchanges a $\mathrm{W}$ with a target neutron to create a proton, and the nucleus remains "intact" throughout the interaction. The neutrino energy in this case can be simply computed by measuring the final state lepton and its outgoing angle with respect to the incoming neutrino direction, which is known. The next process which becomes important as the neutrino energy increases is resonance production, where instead of producing a neutron from a proton, a resonance $(\Delta)$ is produced, which can than decay strongly to a proton and a pion. This process becomes important around about $1 \mathrm{GeV}$. Finally, there is deep inelastic scattering, where the target nucleus is completely broken up, and there are many final state particles, whose energies when summed equal the incoming neutrino energy. One rule of thumb is that all three process contribute about equally to the total cross section at about $1 \mathrm{GeV}$, but above there the DIS cross section rises linearly with neutrino energy while the resonance and quasielastic cross sections remain constant.

Although the previous paragraph discusses charged current interactions where there is at least a final state charged lepton, there are analagous neutral current (NC) interactions, and these can provide significant background when one is searching for $\nu_{\mu} \rightarrow \nu_{e}$. In neutral current interactions the outgoing lepton is a neutrino, and there is often a neutral pion produced, which, depending on the detector, could be mistaken for an electron. Luckily, however, these pions have a steeply falling energy distribution, and because of the missing neutrino energy the total visible energy in the event is less than that of the incoming neutrino energy. Nevertheless, because the probability one is trying to measure may be extremely small, large rejection factors are needed. In order to reduce the neutral current background to a level near that of the intrinsic electron neutrino contamination in the beam, a detector would have to have a rejection factor of about one in 500 .

It is important to point out, however, that although it is straightforward to predict which processes will give a background to these neutrino oscillation experiments, the cross sections for many of those processes are currently far from well-measured. An example of one of the best-measured process that will give backgrounds to conventional oscillation searches is the process $\nu_{\mu} n \rightarrow \mu^{-} p \pi^{0}$, shown in 


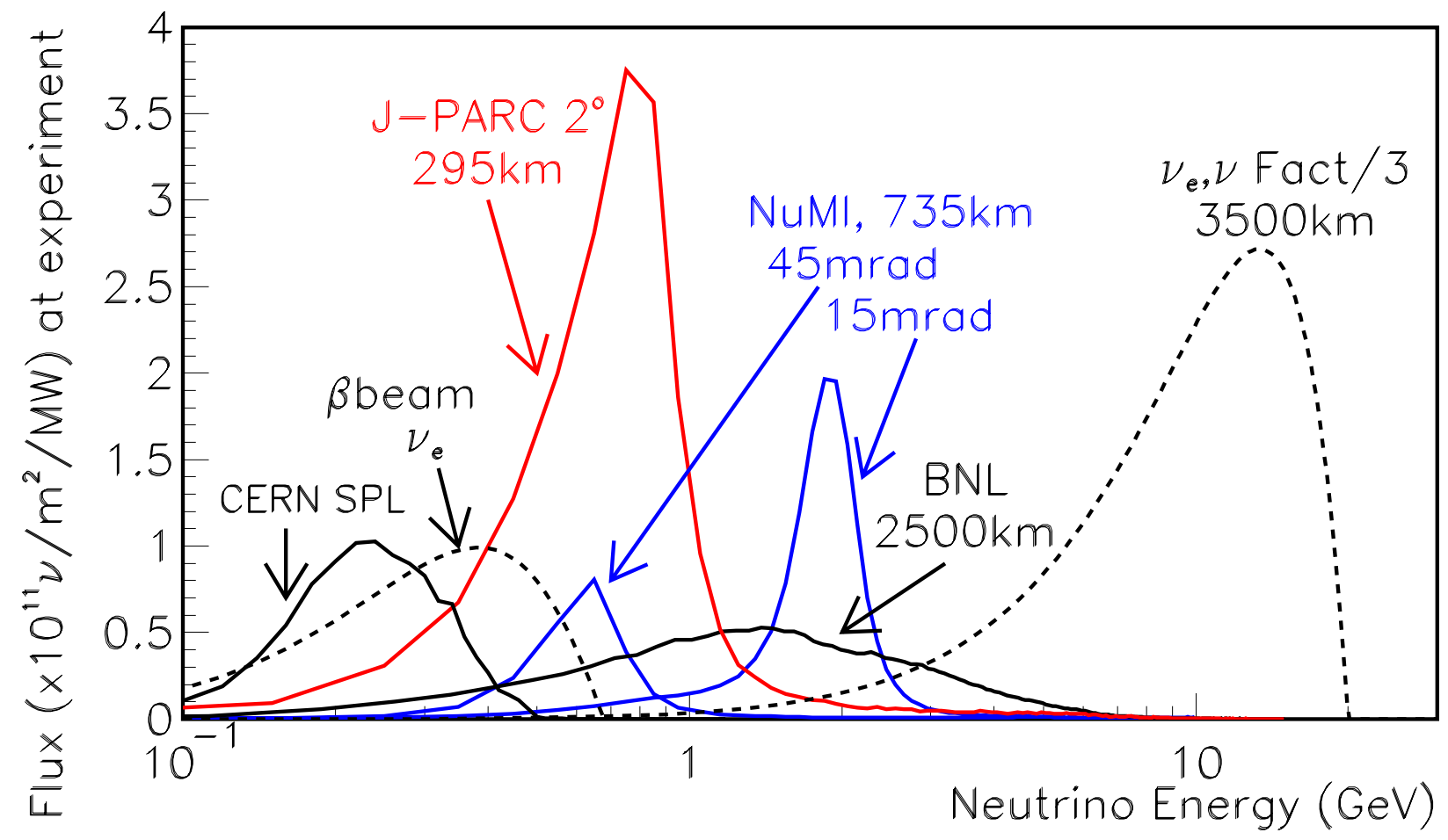

Figure 1. Neutrino fluxes for a variety of beamlines: superbeams, beta-beams,and neutrino factories are shown, where the $\nu_{e}$ fluxes are given in dotted lines, and $\nu_{\mu}$ fluxes in solid lines. The fluxes are normalized to $1 \mathrm{MW}$ proton power, except for the beta-beam, which uses considerably less proton power but is limited by different factors.

figure 2 (see reference ${ }^{9}$ ). This process is extremely important in $\nu_{\mu} \rightarrow \nu_{e}$ searches because detectors can potentially mistake $\pi^{0}$ 's for electrons. It turns out that nuclear effects are important here, and the neutral current analog cross section $\left(\nu_{\mu} n \rightarrow \nu_{\mu} n \pi^{0}\right)$ is even more poorly constrained, since one cannot measure in that case the incoming neutrino energy.

\subsection{Water Cerenkov}

It is largely due to the success of Water Cerenkov devices as neutrino detectors that we know as much as we do about neutrino masses and mixing. Because the neutrino target material (water) is extremely cheap and only the surface of the detector must be instrumented, this technique allows one to afford an extremely massive detector. In fact, the most massive water Cerenkov detector (SuperKamiokande) of today is a factor of ten heavier than the next most massive detector, steel-scintillator (MINOS). Water Cerenkov detectors can identify neutrino interactions through the detection of rings of Cereknov light com-

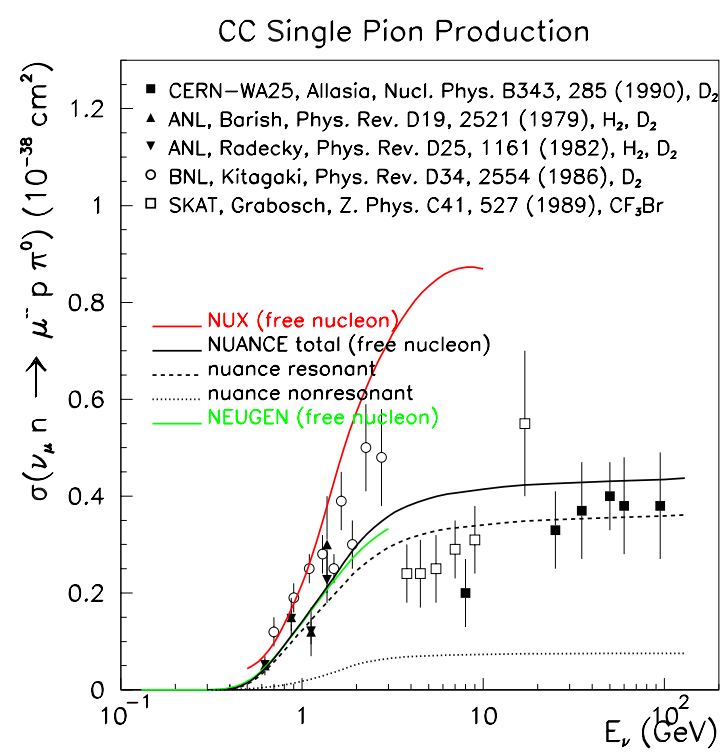

Figure 2. Compilation of cross sections for charged current single pion production, and predictions for different neutrino event generators. Reference is included in the text 
ing from the outgoing charged lepton. The total light measured determines the lepton energy, and the position of the ring determines the outgoing lepton direction, which combined with the lepton energy gives an accurate measurement of the incoming neutrino energy for quasielastic events. Finally, the character of the ring itself can be used for particle identification: a muon scatters very little and therefore leaves a ring with very sharp edges, while an electron scatters much more and makes a ring with "fuzzy" edges, as shown in the upper left event display in figure $3^{10}$.

As the neutrino energy increases, however, and other process begin to dominate the cross section, Water Cerenkov detectors do not measure the total neutrino energy nearly as well. This is because there are other final state particles in the event, for example, charged pions or protons, which are below the cerenkov threshold for water, yet which still carry off a substantial amount of energy. Also, at higher energies, the background rejection is considerably worse. the production of $\pi^{0}$ s from neutral current events is a larger problem. At low energies (about $1 \mathrm{GeV}$ or so), the two rings which come from converted photons from $\pi^{0}$ decays are easily distinguished, and only a small fraction of them overlap 11. However, as the $\pi^{0}$ energy increases, the two photons are emitted close together, and eventually the resulting rings have a very high probability of overlapping.

\subsection{Fine-Grained Calorimetry}

Another technique which has long been in use to detect neutrinos is calorimetry: interleaving planes of absorber with planes of active material. The flavor of the neutrino interacting can be determined by the longitudinal energy deposition of tracks in the event: a muon tends to make a long minimum ionizing track, while an electron makes a much shorter highly ionizing track (see the upper right event display in figure $3^{12}$ ). Since the active material is sensitive to the charge crossing the plane, the thresholds for particle detection can be significantly lower than that of a Cerenkov detector, and depend largely on the segmentation of the passive material. Therefore, fine-grained calorimeters do a good job of measuring the neutrino energy, and in fact can do even better as the final state particles become more energetic and are themselves better measured. As an example, a detector consisting of particle board interleaved every third of a radiation length with readout has been shown to measure neutrino energies to about $15 \%$ at $2 \mathrm{GeV}^{12}$.

The extent to which fine-grained calorimeters can reject neutral current backgrounds depends on the granularity (both transverse and longitudinal) of the detector. A neutral pion will again have two electromagnetic clusters rather than one, and it is unlikely that the two photons would start showering at the same location, which would be the case for a single electron shower. The same fine-grained calorimeter described has been shown in simulations to achieve a $\mathrm{NC}$ background rejection of better than 1 in 500 , in a narrow band $2 \mathrm{GeV}$ neutrino beam.

\subsection{Liquid Argon TPC}

Finally, a detector which could have superior energy resolution and background rejection compared to either of the technologies discussed above is a Liquid Argon Time Projection Chamber, such as is being used by the ICARUS experiment ${ }^{13}$. By instrumenting ultra-pure liquid argon with planes of wires in two dimensions, and reading out the signals induced by ionization as a function of time, one can reconstruct a three-dimensional record of a neutrino interaction. With fine enough wire spacing, this kind of detector produces bubble-chamber like images (see lower right event display in figure $3^{13}$ ), with particle identification through $d E / d x$ measurements and precision tracking. In particular, a $\pi^{0}$ could be rejected from an electron simply by seeing if the first few radiation lengths of track were consistent with one or two highly-ionizing particles.

However, we are just beginning as a field to see how a large scale detector of this technology will perform. A small prototype was placed in the WANF beam during the CHORUS/NOMAD run, and high energy neutrino interactions have been recorded where neutral pions can cleanly be idenfied. Also, a 600 Ton module was tested on cosmic rays for several months in 2001, and the tracks seen there also agree well with the monte carlo predictions. In order to get enough fiducial mass for a next generation experiment, however, we must first see how large a single volume can be made which still functions effectively, and also it is important to study a small prototype in a low energy neutrino beam to again 

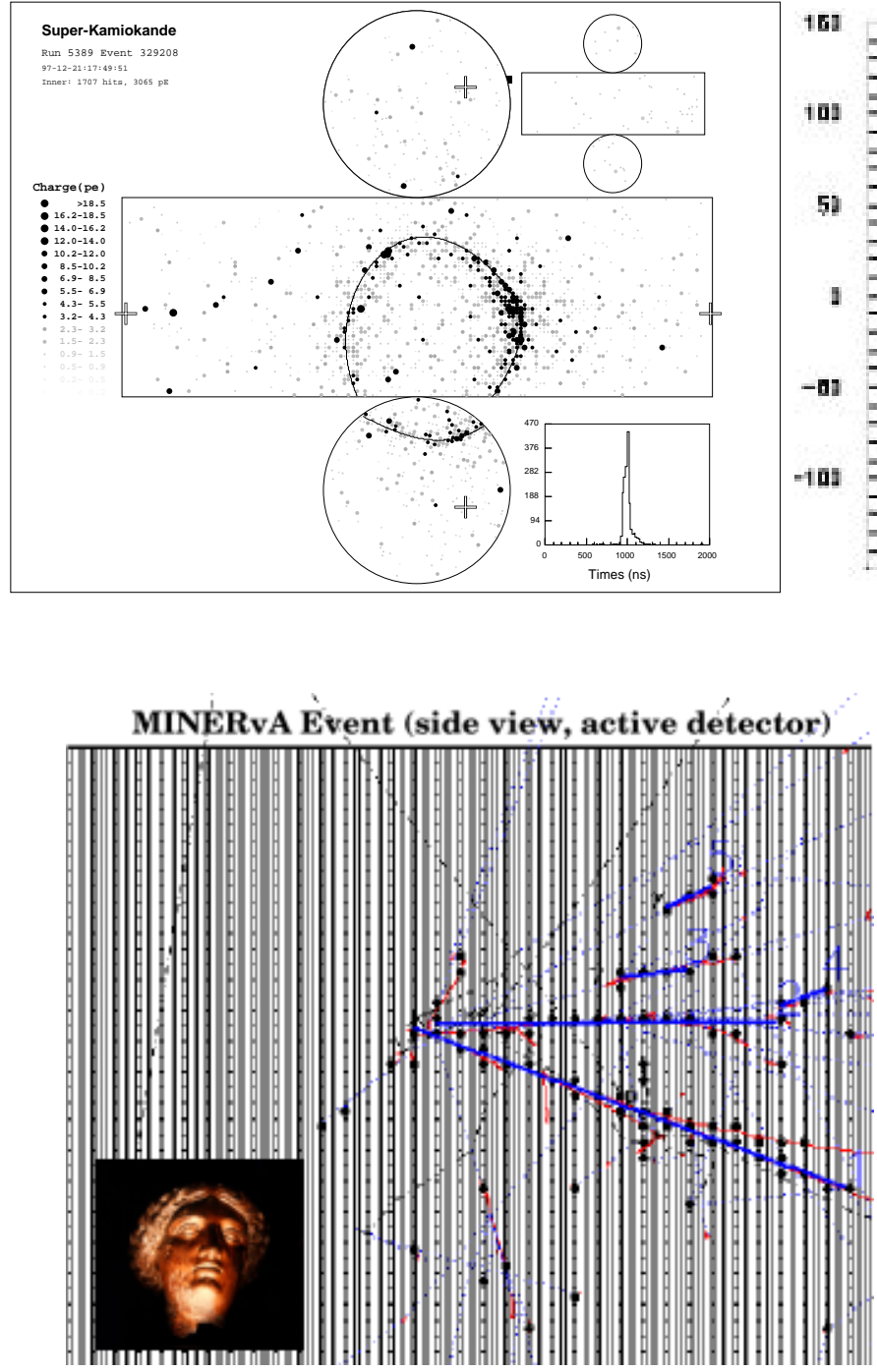
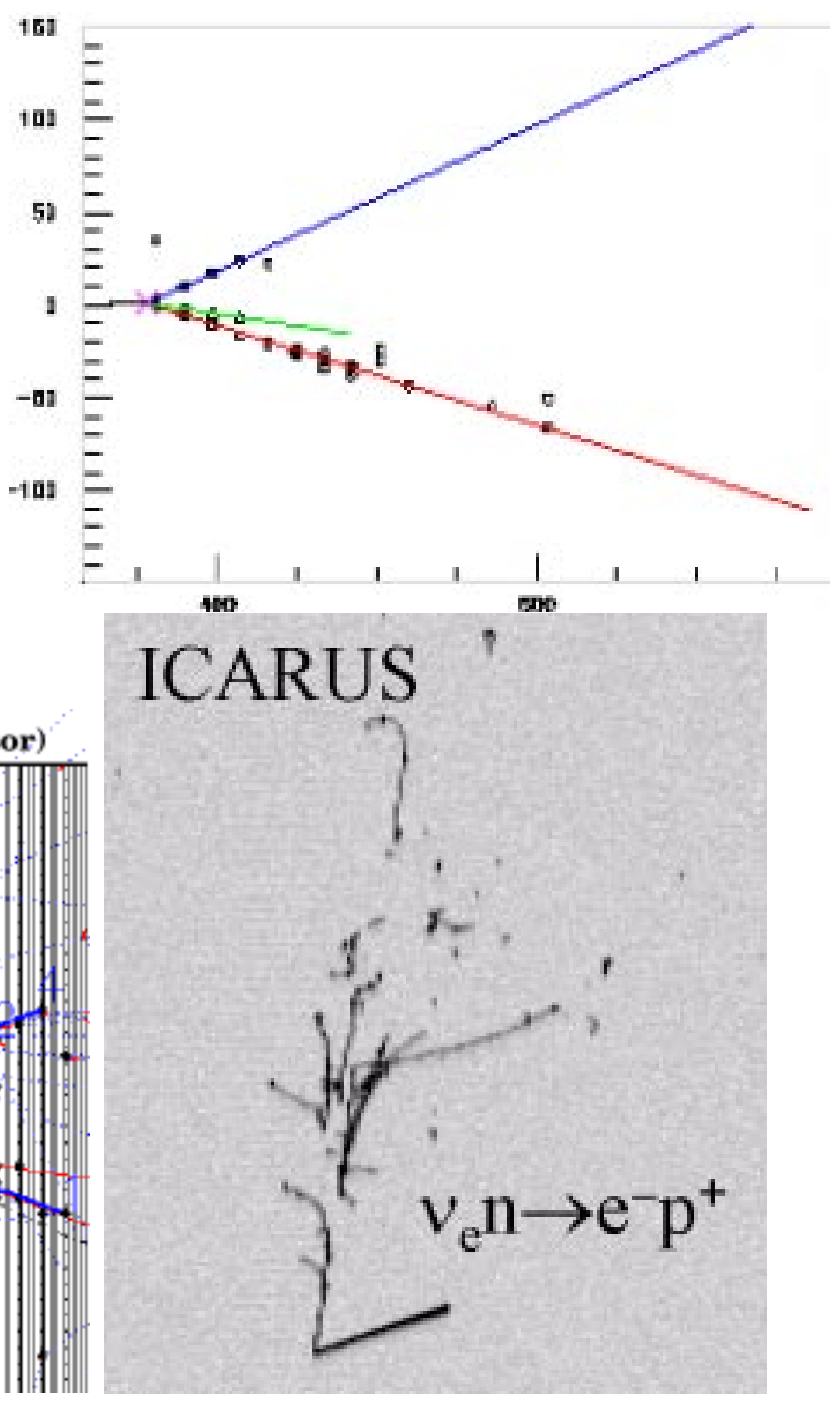

Figure 3. Neutrino event displays for a variety of different detectors: from upper left, clockwise: a $\nu_{e}$ quasielastic event in water Cerenkov, Low-Z calorimeter, Liquid Argon TPC, and finally a potential background event with a $\pi^{0}$ in an all scintillator detector. References given in the text.

confirm the simulations at those energies.

\subsection{Near Detectors}

Figure 3 shows what signal events would look like for a water Cerenkov detector ${ }^{10}$, a fine grained calorimeter ${ }^{12}$, and a liquid argon TPC ${ }^{13}$. The neutral current background is expected to negligible for the liquid argon TPC, but for the other detector technologies this background can be as large as the intrinsic $\nu_{e}$ 's in the beamline. For an accurate far detector prediction one needs not only a near detector measurement of the number of total background events surviving all cuts, but also an understanding of how that number changes when extrapolating to the far detector. To do this, we as a field need accurate dedicated cross section experiments, using detectors which can do more than simply identify an event as a signal candidate. The K2K experiment has initiated this program by their suite of near detectors at KEK, and at this conference K2K has presented an updated measurement of the ratio of $\pi^{0} / \mu$ production ${ }^{11}$. Another example being proposed, which would operate at much higher neutrino energies is the Minerva experiment ${ }^{14}$, which would use the NuMI Beamline at Fermilab with a fully active scintillator target. A sample event display from a potential 
background source ( $\pi^{0}$ production $)$ is also shown in figure 3. Clearly by measuring much more about the various processes we can vastly improve our models of neutrino interactions, and then the predictions for a far detector can be made accurately.

Although it will not be stated explicitly, each of the following experiments being described will plan to have at least one near detector in their beamline, and in some cases more than one.

\section{Determining the Number of Neutrinos}

The first question that must be answered before we really understand what future experiments can do, is whether or not the appearance of $\bar{\nu}_{e}$ 's in a $\bar{\nu}_{\mu}$ beam at Los Alamos is due to oscillations or not. If MiniBooNE confirms the LSND neutrino signal, then there is much more to measure and many more basic questions that must be asked than what is usually described in future experiment proposals.

One example of a new signature which could be implied if MiniBooNE sees a signal, is a sizable disappearance probability at the "LSND $\Delta m^{2}$ ". However, in order to measure the disappearance to the required precision, MiniBooNE would have to add a second detector to the beamline. The optimal location for that second detector depends on what $\Delta m^{2}$ is actually measured by MiniBooNE.

Another implication if there are sterile neutrinos is that there could be sizable CP-violating effects in the $\nu_{\mu} \rightarrow \nu_{\tau}$ channel and or the $\nu_{e} \rightarrow \nu_{\mu}$ channel, again at the "LSND $\Delta m^{2} " 15$. There are also models where CPT is violated, and the disappearance signature at the atmospheric $\Delta m^{2}$ would be different between neutrinos and antineutrinos ${ }^{16}$.

It is clear that independent of the theories that are being considered today, if MiniBooNE confirms the LSND signature to be due to neutrino oscillations then there is much more to do than is even outlined here. In the interest of brevity, the remainder of this document will make the assumption that this does not happen.

\section{Near Term $\theta_{13}$ Experiments: Phase I}

The two experiments described next appear at first glimpse to be rather similar: they are both optimized to search for $\nu_{\mu} \rightarrow \nu_{e}$ in a conventional beam, and expect to run near the first oscillation maximum, or where $\Delta m_{23}^{2} L / 4 E$ is about $\pi / 2$. To do this they both use off-axis neutrino beams, and both take advantage of significant investments in neutrino physics that have already been made. The experiments themselves are first described, but then we consider what could be learned by having both measurements, in the case where a signal is seen.

\subsection{J-PARC to SuperKamiokande}

A new hadron facility is being constructed at Jaeri, called J-PARC ${ }^{17}$. It will have a $0.8 \mathrm{MW}$ proton source, with an initial proton energy of $40 \mathrm{GeV}$ which could eventually be raised to $50 \mathrm{GeV}$. Connected with that facility is a neutrino beamline, which would be used in conjunction with the SuperKamiokande Water Cerenkov detector ${ }^{18}$. By using the off axis technique and also building a decay pipe that is trapezoidal in shape, this experiment has the flexibility to run at several different off axis angles, which correspond to different neutrino energies, ranging from a $550 \mathrm{MeV}$ beam to a $700 \mathrm{MeV}$ beam. The resulting electron neutrino background is roughly $0.2 \%$, at the muon neutrino peak, and slighly higher when integrated over the entire width of the peak.

The first stage of this experiment expects to be sensitive to values of $\theta_{13}$ up to about a factor of 10 past the $\mathrm{CHOOZ}$ limit, after 5 years of running. The experiment has recently been included in Phase I of the J-PARC running, and hopes to start taking high intensity neutrino data in 2008. The construction of the neutrino beamline is expected to begin in 2004, and of course, the SuperKamiokande detector is already in place and fully functional.

A later stage, which involves a more powerful proton source and a larger detector, is also under consideration. This stage will focus on $\mathrm{CP}$ violation and therefore have to run for significant periods in "antineutrino" mode.

\subsection{NuMI Off Axis Experiment}

While the NuMI beamline is busy making an on axis neutrino experiment to measure $\Delta m_{23}^{2}$ (MINOS), it will also be producing "off axis" neutrino beams at sites all over northern Minnesota and southern Ontario $^{12}$. If one were to place a detector at roughly $20 \mathrm{mrad}$ from the NuMI beamline, it would see a very narrow-band beam at $2 \mathrm{GeV}$. At $40 \mathrm{mrad}$ from the beamline, the narrow-band beam is closer to $1 \mathrm{GeV}$. 
Because the NuMI beamline will only be getting protons (and hence making neutrinos) for 10 microseconds out of every 2 seconds, it is possible to consider placing a detector at the surface of the earth, and using timing to reject cosmic ray backgrounds. Because one is not constrained by needing a large underground cavern, there is a huge flexibility of experiments that can be proposed: one can vary both the energy and the baseline, somewhat independently, by simply changing the off axis angle.

A first stage of a NuMI off-axis experiment is being proposed, and involves placing a fine-grained calorimeter at a distance of about $800 \mathrm{~km}$ from the neutrino source at Fermilab, and at an off axis distance of $10 \mathrm{~km}$, corresponding to about $12.5 \mathrm{mrad}^{12}$. At this location the electron neutrino background is $0.5 \%$ when integrated over the peak, which is itself about $20 \%$ FWHM. For a neutrino beam of this energy and baseline, the matter enhancement (or suppression) is about $20 \%$.

A later stage of this experiment would also involve running in antineutrino mode with an upgraded proton source, but also there is the flexibility to run at different off axis angles and baselines, depending on what the first generation sees.

\section{When the Whole is greater than the sum of its parts}

In order to understand the motivation for building two long baseline neutrino experiments rather than simply running one of them for twice as long (or building it to be twice as powerful), it is helpful to look at the oscillation probability to all orders ${ }^{19}$. One useful expansion of the full probability in the earth is as follows ${ }^{20}$ :

$$
\begin{aligned}
& P=P_{1}+P_{2}+P_{3}+P_{4} \\
& P_{1}=\sin ^{2} \theta_{23} \sin ^{2} 2 \theta_{13}\left(\frac{\Delta_{13}}{B_{ \pm}}\right)^{2} \sin ^{2} \frac{B_{ \pm} L}{2} \\
& P_{2}=\cos ^{2} \theta_{23} \sin ^{2} 2 \theta_{12}\left(\frac{\Delta_{12}}{A}\right)^{2} \sin ^{2} \frac{A L}{2} \\
& P_{3}=J \cos \delta\left(\frac{\Delta_{12}}{A}\right)\left(\frac{\Delta_{13}}{B_{ \pm}}\right) \cos \frac{\Delta_{13} L}{2} \sin \frac{A L}{2} \sin \frac{B_{ \pm} L}{2} \\
& P_{4}=\mp J \sin \delta\left(\frac{\Delta_{12}}{A}\right)\left(\frac{\Delta_{13}}{B_{ \pm}}\right) \sin \frac{\Delta_{13} L}{2} \sin \frac{A L}{2} \sin \frac{B_{ \pm} L}{2}
\end{aligned}
$$

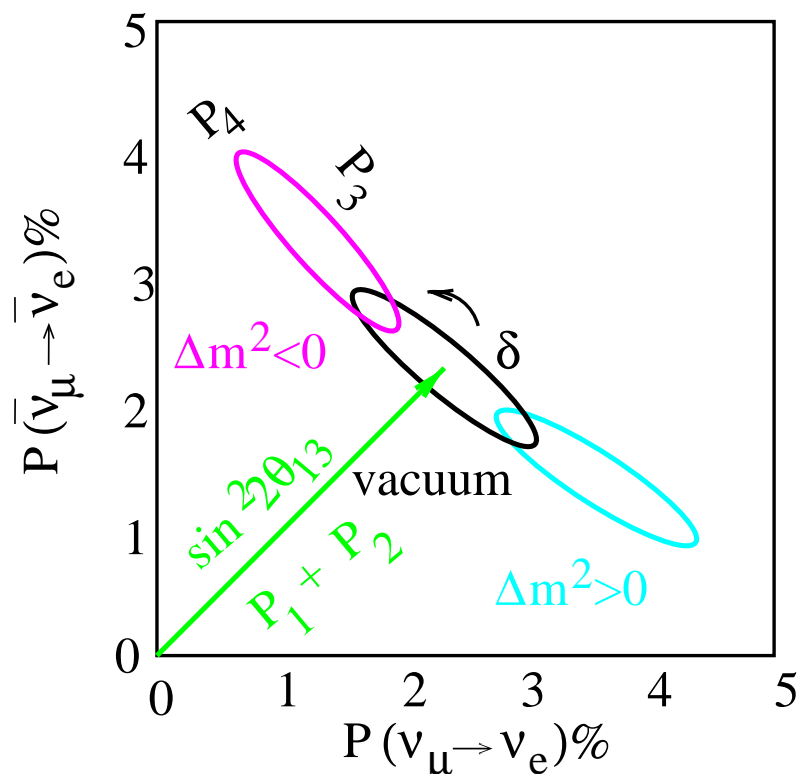

Figure 4. Neutrino versus Antineutrino Probabilities for $\nu_{\mu} \rightarrow$ $\nu_{e}$, at a given baseline and neutrino energy, for a single value of $\sin ^{2} 2 \theta_{13}$

where

$$
\begin{aligned}
\Delta_{i j} & =\frac{\Delta m_{i j}^{2}}{2 E_{\nu}} \\
A & =\sqrt{2} G_{F} n_{e} \\
B_{ \pm} & =\left|A \pm \Delta_{13}\right| \\
J & =\cos \theta_{13} \sin 2 \theta_{12} \sin 2 \theta_{13} \sin 2 \theta_{23}
\end{aligned}
$$

and the \pm signifies neutrinos or antineutrinos

As an example of the ambiguities associated with one neutrino and antineutrino measurement, all the possible values of the neutrino and antineutrino probabilities for one single value of $\theta_{13}$ at one experiment are shown in figure 4 . So although one measurement of neutrino oscillation probablity can set a lower limit on how large $\theta_{13}$ is, it is far from a determination of the mixing angle. It has been shown that even with a neutrino and antineutrino measurement at one energy and baseline, there can be several solutions of $\theta_{13}$ and $\delta$, because of imperfect knowlege of the mass hierarchy, or how far $\sin ^{2} 2 \theta_{23}$ is from $\pi / 4$ 21 .

So clearly, to get to the remaining underlying physics, namely the mass hierarchy and $\mathrm{CP}$ violation in the lepton sector, we need more than one neutrino and one antineutrino measurement of $\nu_{\mu} \rightarrow \nu_{e}$. We must measure both these transitions at least once but preferably at more than one energy, and one of the 
sets of measurements must be over a large enough baseline that the matter effects in the earth are significant (at least $700 \mathrm{~km}$ ).

\section{Phase II Experiments: The next decade in $\sin ^{2} 2 \theta_{13}$}

What kinds of experiments are done in the longer term future depend very much on what the first generation of experiments sees. One can imagine several scenarios, depending on which experiment sees evidence for $\theta_{13}$ first. It is helpful to group them in ranges of $\sin ^{2} 2 \theta_{13}$, where for each range a different set of experiments may be able to make the relevant measurements. Table 1 shows for different ranges of $\sin ^{2} 2 \theta_{13}$, which experiments (or combinations or experiments) can do which physics.

Consider the discovery of $\nu_{\mu} \rightarrow \nu_{e}$ : if $\sin ^{2} 2 \theta_{13}$ is within a factor of 2 of the CHOOZ limit, the MINOS or CNGS experiments should be able to get the first evidence. If $\sin ^{2} 2 \theta_{13}$ is not seen in MINOS or CNGS, but is within a factor of roughly 10 of the CHOOZ limit, the NuMI-Off Axis and/or J-PARC would be able to see the first evidence. Then, if it is not seen at these next generation experiments, then upgrades to either NuMI Off Axis or J-PARC to SuperK would be recommended (Phase II). Recall that to reach another factor of 10 improvement in the limit, however, one must increase the product of the proton power and the detector mass by a factor of 100! Finally, if they are not seen at any of those next generation experiments, one would turn to a neutrino factory where still another factor of 10 or 100 in $\sin ^{2} 2 \theta_{13}$ could be accessed. The first row of table 1 shows this progression.

But of course there is much more to be learned than simply determining that $\theta_{13}$ is non-zero. If it is discovered in MINOS or CNGS, then NuMI Off-Axis and J-PARC experiments combined have a chance of seeing either the mass hierarchy, or a hint of $\mathrm{CP}$ Violation, if it is maximal, as shown in Figure 5), and described in references ${ }^{2223}$ and more generally in the collection of references in note ${ }^{19}$. This is represented by the first column of table 1 .

If it is discovered in J-PARC to SuperK or NuMI Off-Axis, then there are a host of Phase II experiments being proposed which would have a chance of seeing $\mathrm{CP}$ violation and determining the mass hierarchy in a broader range of parameter space than

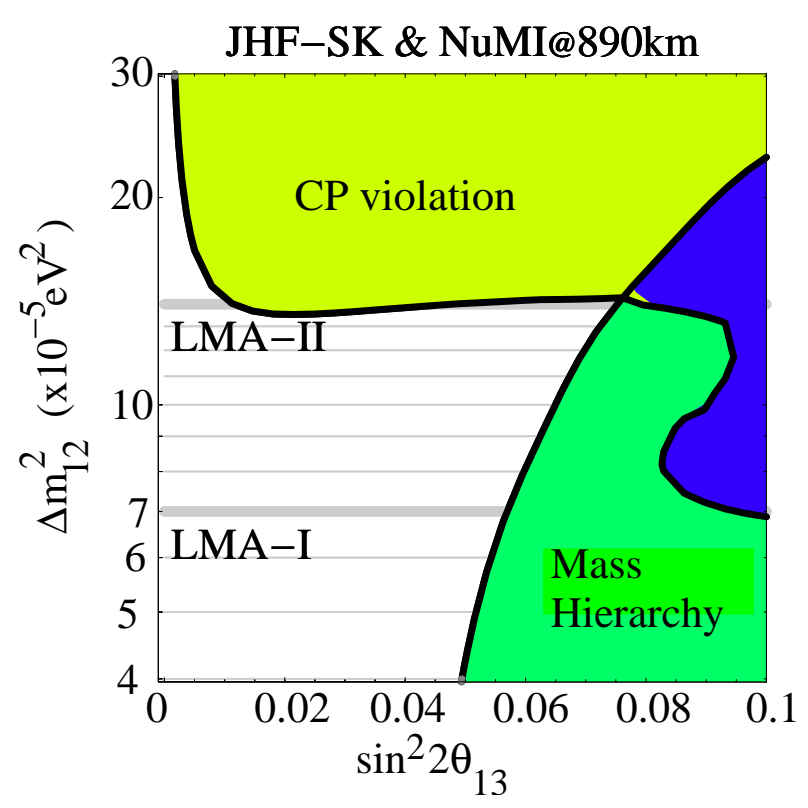

Figure 5. Ranges in $\Delta m_{12}^{2}$ and $\sin ^{2} 2 \theta_{13}$ parameter space where maximal $\mathrm{CP}$ violation or the mass hierarchy could be determined. Reference given in text

what is shown in figure 5. Those experiments are described in the following section, and would appear in the second column of table 1.

\subsection{Upgrades to J-PARC or NuMI Off Axis}

In order to get a substantial improvement in the reach of any of the experiments described so far, one must improve not only the proton source, but also the detector mass. Of course it is only the product of the two which determines the statistics (and hence sensitivity) of the experiment, but the costs of improving either by itself are far from linear. For example, beamlines are currently in use which could withstand about 1MW of power, but much work must still be done to understand what kinds of targets could withstand $4 \mathrm{MW}$ of proton power. Some of this work is already being carried out by the muon storage ring collaboration, because this is the same power that is needed for a neutrino factory. Similarly, although one could "simply" build 10 Superkamiokande detectors, and the cost there would scale roughly linearly, there is some gain to be made by making the detector volume larger. Since one is only instrumenting the surface of the detector, and the dominant cost of the detectors is in the phototubes, the cost would (ideally) go only as 
Table 1. Summary of which experiment can contribute to which decade of $\sin ^{2} 2 \theta_{13}$

\begin{tabular}{|c|c|c|c|c|}
\hline \multirow[b]{2}{*}{ Physics } & \multicolumn{4}{|c|}{ Value of $\sin ^{2} 2 \theta_{13}$} \\
\hline & $>4 \times 10^{-2}$ & $>1 \times 10^{-2}$ & $>\times 10^{-3}$ & $>\times 10^{-4}$ \\
\hline seeing $\theta_{13} \neq 0$ & $\begin{array}{l}\text { MINOS } \\
\text { CNGS }\end{array}$ & $\begin{array}{l}\text { Conventional } \\
\text { Superbeams } \\
\text { Phase I }\end{array}$ & $\begin{array}{c}\text { Conventional } \\
\text { Superbeams } \\
\text { Phase II }\end{array}$ & $\begin{array}{c}\nu \text { Fact } \\
L \geq 3500 k m\end{array}$ \\
\hline $\begin{array}{l}\text { Mass } \\
\text { Hierarchy }\end{array}$ & $\begin{array}{l}\text { Combinations } \\
\text { of Phase I } \\
\text { Superbeams }\end{array}$ & $\begin{array}{c}\text { Combinations } \\
\text { of Phase II } \\
\text { Super/beta Beams }\end{array}$ & $\begin{array}{c}\text { Combinations } \\
\text { of } \nu \text { Factory } \\
\text { and Super/beta beams }\end{array}$ & $\begin{array}{c}\nu \text { Factory } \\
L \sim 7700 \mathrm{~km}\end{array}$ \\
\hline $\begin{array}{l}\text { Evidence for } \\
\text { CP violation }\end{array}$ & $\begin{array}{l}\text { Combinations } \\
\text { of Phase I } \\
\text { Superbeams }\end{array}$ & $\begin{array}{c}\text { Combinations } \\
\text { of Phase II } \\
\text { Super/beta Beams }\end{array}$ & $\begin{array}{c}\text { Combinations } \\
\text { of } \nu \text { Factory } \\
\text { and Super/beta beams }\end{array}$ & $\begin{array}{l}\text { Combinations } \\
\text { of } \nu \text { Factory } \\
2 \text { baselines }\end{array}$ \\
\hline
\end{tabular}

the $2 / 3$ power of the mass. Also, for larger detectors, the ratio of fiducial volume to total volume is closer to 1: it is worth noting that although the SuperKamiokande detector is 50kton, the fiducial volume is only $22.5 \mathrm{kton}$, less than half.

Although the target design is not understood, the remainder of the J-PARC to SuperK beamline is being constructed to be able to withstand a factor of 8 increase of proton power above what is assumed for Phase I. The proton source itself would have to be increased by that factor of 8 , however. An upgrade of the detector, called HyperKamiokande, is now being studied. This detector would have a factor of 20 more mass than the SuperKamiokande detector, or 1MTon of water. The HyperKamiokande detector would be located in the same mountain range as the SuperKamiokande detector, and in order for both detectors to see the same range of neutrino beam energies (off axis), the beamline itself points into the earth at a higher angle than would be required for an on axis beam at either of those two locations. Currently there are new technologies for photo-sensors which are being studied, since they not only dominate the cost, but also would need to be more robust, since the detector volume (and hence water pressure!) is increasing in future proposals ${ }^{24}$.

Similarly, in order to produce more neutrinos in the NuMI Beamline, the Fermilab proton source would need to be upgraded. There are currently two designs for an upgraded Booster, which would provide $8 \mathrm{GeV}$ protons that then get accelerated in the Main Injector ${ }^{25}$. One is an upgraded proton synchrotron which is similar in size to the current Booster, and the other is a proton linac which would use very novel acceleration techniques which are of interest to the linear collider community. Either of those designs, combined with a modest upgrade of the Main Injector and the NuMI Beamline itself, could bring the NuMI facility up to $2 \mathrm{MW}$, or a factor of 5 above what the first generation experiment hopes to see. The way in which the NuMI Off-Axis detector would be upgraded depends slightly on what the first generation of experiments finds. If CP violation and Matter effects both act in the same direction to make the neutrino and antineutrino probabilities very different from one another, then the best bet would be to add more detector at the same location-either by copying the existing design, or upgrading with a more sensitive detector with better background rejection (for example, liquid Argon).

However, if the first generation of the NuMI Off Axis experiment sees virtually the same neutrino and antineutrino probabilities, this means the $\mathrm{CP}$ violating effects and matter effects are cancelling one another. In order to make progress one would use a different location-for example, by going to the second appearance maximum (at for example, the same baseline but one third the neutrino energy as the first experiment) the $\mathrm{CP}$ violating effects would be a factor of 3 larger, but the matter effects would be a factor of 3 smaller. And in this case, by combining both first and second generation results not only could the mass hierarchy be determined, but also there is a chance of seeing maximal $\mathrm{CP}$ violation ${ }^{26}$.

\subsection{Brookhaven Proposal}

In contrast to the proposals described thus far, there is a proposal from a Brookhaven-based study group that aims to use one broad band neutrino beam at an extremely long distance ${ }^{27}$. This experiment proposes that the Brookhaven AGS, which produces $28 \mathrm{GeV}$ protons, be upgraded to $1 \mathrm{MW}$, where targeting is 
still manageable with today's technology. The detector for this proposal would be a very large underground water cerenkov device, which would presumably be part of a National Underground Science Laboratory. One design for this detector, the UNO detector ${ }^{28}$ would have a fiducial mass of $450 \mathrm{kton}$, a factor of 20 over the superKamiokande fiducial mass.

Because the signal distribution is now spread over a broad energy region, the experiment must take particular care in improving the signal to background ratio. The first step is to reduce the background by using only single-ring (quasi-elastic-like) events for all neutrino energies, since $\pi^{0}$ 's tend to make two rings. The second step is to increase the signal probability-this is done in two ways: at high energies, the matter effects will enhance the probability of either neutrinos or antineutrinos many times above what it would be at low energies. At lower energies in this experiment, the baseline is so long that these neutrinos are at the second and third oscillation maxima, where the CP-violating effects are 3 or 5 times as large. Although the experiment would start in neutrino running, if at first the evidence for $\nu_{\mu} \rightarrow \nu_{e}$ is not seen at high energies, then they would switch to antineutrino running where the matter enhancement would then be important.

Because of the broad band neutrino beam, the experiment would have a sensitivity to more than just maximal CP-violation. If $\delta$ is close to 0 , then the $\cos \delta$ terms in the probability become important, and those can contribute at the oscillation mimina, or at energies where $\Delta m^{2} L / 4 E$ is equal to $\pi$ or $2 \pi$.

\subsection{CERN SPL and BetaBeams}

There is a proposal based at CERN which is focused not only on CP violation measurements with a very low energy conventional beamline, but also on $\mathrm{T}$ violation by comparing muon appearance from an electron neutrino beam with the conventional neutrino beam results ${ }^{29}$. The intent is to build two beamlines, one conventional, which uses the $2.2 \mathrm{GeV}$ protons from the CERN SPS, and one beta-beam, which uses the ISOL technology, and pointing them to the same far detector. This plan would take advantage of the extra mountain tunneling that is currently planned in Frejus, and again a very large water Cerenkov detector is being used for the proposal. Because the neutrino cross section at these energies is extremely small, the plan is to start with a $4 \mathrm{MW}$ proton source, and again a detector with 450kton of fiducial mass.

At extremely low neutrino energies (some few hundred $\mathrm{MeV}$ ) the neutral current background is low not only because the relevant cross section ratio $\left(\pi^{0} / \mu\right)$ is lower than at higher energies, but also because the $\pi^{0}$ 's that are produced are so low in energy that when they decay to two photons, they virtually always make two distinct rings.

One downside of these very low energies, however, is that the antineutrino to neutrino cross section ratio is even lower than a third, which is the ratio at $1 \mathrm{GeV}$. Therefore, the sensitivities quoted for this experiment are gained by running for 2 years in neutrino mode and an additional 8 years in antineutrino mode for a conventional beam, with a similar total running time for the beta beam.

Finally, for a $130 \mathrm{~km}$ baseline the matter effects are extremely tiny, and as such do not affect the precision with which this experiment might be able to see $\mathrm{CP}$ violation. However, by the same token this experiment by itself would not be able to determine the mass hierarchy.

\section{The last decade in $\sin ^{2} 2 \theta_{13}$ : neutrino factories}

Once again, the need for the next generation of neutrino experiments changes as a function of what the previous generation has seen. If $\theta_{13}$ is so small that it has just barely been seen by the second generation J-PARC or NuMI Off-axis experiments, then it will take a neutrino factory with a baseline of (very roughly) $3500 \mathrm{~km}$ to adress the question of the neutrino mass hierarchy and CP violation (as represented by the third column in table 1 ). If $\theta_{13}$ is too small to be seen even with conventional beams, then a neutrino factory, running with a $4 \mathrm{MW}$ proton source and a 50kton magnetized detector (such as the design used by MINOS), would still be able to push the sensitivity of $\sin ^{2} 2 \theta_{13}$ another two orders of magnitude. There have been many studies over the past 5 years addressing the capabilities of neutrino factories: reference ${ }^{30}$ describes many of the early ones, and references ${ }^{19}$ are more recent articles describing the tricky business of getting from measured probabilities to the mixing angles themselves.

One strategy to get the farthest reach on $\theta_{13}$ 
was obtained by looking carefully at the probability shown in equation 6 . If one were to design an experiment such that $\sin (A L / 2)$ is equal to 0 , in other words, $A L / 2=2 \pi$, then only the first term $P_{1}$ would contribute to the oscillation probability, and the matter enhancement would be enormous ${ }^{31}$. This condition is satisfied for all energies and $\Delta m^{2}$ values, for a baseline of about $7700 \mathrm{~km}$, and depends only on the density of electrons in the earth. Of course, building a storage ring with such a steep slope will be challenging from a civil engineering standpoint, but the detector technology is extremely straightforward. For this experiment, it is enough to measure one probability-if oscillations are seen at this baseline in $\nu_{e}\left(\mu^{+}\right)$running then one has determined the mass hierarchy and measured $\sin ^{2} 2 \theta_{13}$ with one measurement. If it is not seen then one would switch to antineutrinos (with $\mu^{-}$'s in the ring), where again a large probability would be predicted, even for small values of $\theta_{13}$.

Although we will not know the primary motivation for a neutrino factory until the next generation of experiments runs, we know now that it has the potential to provide the most precise measurements of $\theta_{13}$ and the CP-violating phase, or possibly even the only measurement of $\theta_{13}$ and the mass hierarchy. For this reason alone it is important to continue $R \& D$ to understand how to build a neutrino factory. Since the last time this conference was held there has been much progress in designing a neutrino factory that the field can afford. There have been advances in design of phase rotation, cooling, and acceleration, such that the current design can boast of substantial savings in all of the cost drivers in the facility ${ }^{32}$.

\section{Conclusions}

Table 2 shows the various experiments that have been described in this report. They vary widely in terms of physics reach, time scales for construction and completion, and technical feasibility. Although it is not clear today which path this field will ultimately take, there are two extremely good near term opportunities for the next generation of measurements: both the J-PARC to SuperKamiokande and the NuMI Off Axis proposals should see evidence for $\sin ^{2} 2 \theta_{13}$ if it is within a factor of ten of the current $\mathrm{CHOOZ}$ limit. Once that first evidence is seen then the debate can truly begin on how to best de- sign a program to get to the neutrino mass hierarchy and $\mathrm{CP}$ violation in the lepton sector. The experimental challenges for both accelerator and particle physicists are high but the rewards for these measurements will be higher still.

\section{Acknowledgments}

This report describes the work of hundreds of people working all over the world to figure out how we can get to this exciting physics. I am indebted to them, and in particular to the following people for their help in putting together this presentation: Milind Diwan, Gary Feldman, Steve Geer, Atsuko Ichikawa, Yoshi Kuno, Ken Long, Kevin McFarland, Mauro Mezzetto, Ko Nishikawa, Bob Palmer, Brett Viren, and Walter Winter.

\section{References}

1. M. Apollonio et al., Phys.Lett.B466 415, 1999

2. W.J. Marciano, hep-ph/0108181, August 2001

3. S.Parke first derived this for a talk at WIN2003, October 2003.

4. P. Zucchelli, Phys.Lett.B532, 166, 2002

5. S.Geer, Phys.Rev. D57 6989, 1998

6. M. Lindroos et al., CERN/PS-2002-078 (2002).

7. J. Bouchez, presented at NuFact03, 2003.

8. C. Albright et al (Eds. S. Geer and H. Schellman), Report to the Fermilab Directorate FERMILAB-FN692, April 2000, hep-ex/0008064

9. See G.P.Zeller, talk given at NuINT02 for a summary of the current status of low energy neutrino cross section data.

10. "Evidence for Neutrino Mass from Observations of Atmospheric Neutrinos with Super-Kamiokande", dissertation of Mark Messier, Boston University, 1999

11. K. Nishikawa, Lepton Photon transparencies and proceedings.

12. D. Ayres et al, hep-ex/0210005 and http: //www-off-axis.fnal.gov

13. Updated Icarus Technical Design Report, CERN/SPSC 2002-027, see also http://pcnometh4.cern.ch/ http://budoe.bu.edu/ ${ }^{\text {messier/thesis/ }}$

14. See http://www . pas. rochester.edu/minerva

15. V.Barger, S.Geer, R.Raja, K.Whisnant, et al, Phys. Rev. D63, 033002, 2001

16. G.Barenboim, J.Lykken, Phys. Lett. B554 73, 2003

17. J-PARC web page: http://neutrino.kek.jp/jhfnu/

18. Itow et al, J-PARC to SuperKamiokande Letter of Intent, hep-ex/0106019

19. V. Barger, D.Marfatia, K.Whisnant, Phys. Lett. B560 75, 2003, and K. Dick, M. Freund, P. Huber, M. 
Table 2. Summary of various experiments described in this report.

\begin{tabular}{|c|c|c|c|c|c|c|c|}
\hline $\begin{array}{l}\text { Beam } \\
\text { Name }\end{array}$ & $\begin{array}{c}\text { Peak Energy } \\
(\mathrm{GeV})\end{array}$ & $\begin{array}{c}\text { Baseline } \\
(\mathrm{km})\end{array}$ & $\begin{array}{l}\text { Mass } \\
\text { (kton) }\end{array}$ & $\begin{array}{l}\text { Power } \\
\text { (MW) }\end{array}$ & $\begin{array}{c}\sin ^{2} 2 \theta_{13} \\
\text { sens. }{ }^{a}\end{array}$ & $\delta^{b}$ & $\begin{array}{l}\text { Matter } \\
\text { Effect }\end{array}$ \\
\hline OPERA $^{33}$ & 17 & 732 & 1.8 & 0.15 & 0.04 & - & \\
\hline ICARUS $^{33}$ & 17 & 732 & 2.4 & 0.15 & 0.03 & - & \\
\hline MINOS $^{34}$ & 3.5 & 735 & 5 & 0.4 & 0.05 & - & \\
\hline $\mathrm{CNGS}^{35}$ & 2 & 732 & 2.35 & .15 & $\sim 0.02^{* *}$ & & $\geq \mathrm{CP}$ \\
\hline JHF2SK & 0.8 & 295 & 22.5 & 0.8 & 0.006 & - & - \\
\hline NuMI-OA & 2 & 850 & 50 & 0.4 & 0.004 & - & $\geq \mathrm{CP}$ \\
\hline SJHF2HK & 0.8 & 295 & 450 & 4 & $\sim 0.001^{s}$ & $|\delta|>20^{\circ}$ & $<\mathrm{CP}$ \\
\hline SNUMI-OA & 1 or 2 & $>800$ & 100 & 2 & $\sim 0.001^{s}$ & $135 \pm 20$ & $\geq \mathrm{CP}$ \\
\hline BNL2NUSL & 1.5 & $>2500$ & 500 & 1 & $\begin{array}{c}0.004 \\
\mathrm{CP}\end{array}$ & $45 \pm 20$ & $>\&<$ \\
\hline CERN SPL & 0.2 & 130 & 400 & 4 & 0.0016 & $90 \pm 30$ & $\ll \mathrm{CP}$ \\
\hline$\beta$ Beam & 0.2 & 130 & 400 & .04 & & $\mathrm{~T}$ viol. & $\ll \mathrm{CP}$ \\
\hline$\nu$ Factory & 15 & $\sim 3000$ & 50 & 4 & few $\times 10^{-4}$ & $90 \pm 20$ & $\gg \mathrm{CP}$ \\
\hline$\nu$ Factory & 15 & $\sim 7700$ & 50 & 4 & $<10^{-4}$ & - & huge \\
\hline
\end{tabular}

Lindner, Nucl. Phys. B598 543, 2001, and J. BurguetCastell, M.B.Gavela, J.J. Gomez-Cadenas, P. Hernandez, O. Mena, Nucl.Phys. B646 301,2002

20. H. Minakata, H. Nunokawa, JHEP 0110, 001, 2001

21. V. Barger, D.Marfatia, K.Whisnant,Phys.Rev.D65, 073023,2002

22. P. Huber, M. Lindner, W. Winter, Nucl.Phys.B654 3, 2003

23. H. Minakata, H. Nunokawa, S. Parke Phys. Rev. D68, 013010, 2003

24. K. Nakamura, talk at Neutrinos and Implications for Physics Beyond the Standard Model, Stony Brook, October 2002, http://insti.physics.sunysb.edu/ itp/conf/neutrino.html

25. Proton Driver Design Study http://www-bd.fnal.gov/pdriver/8GEV

26. G. Feldman, July 3 Hepap Meeting, http://doe-hep.hep.net/HEPAP/Agendajuly03.html

27. M.V.Diwan et al hep-ph/0303081 March 2003, M.V.Diwan et al hep-ex/0211001 October 2002, and D.Beavis et al, hep-ex/0205040, April 2002

28. UNO whitepaper,"Physics Potential and Feasibility of UNO", June 2001 SBHEP01-3 available at http://superk .physics . sunysb.edu/uno/

29. The physics potential of both the CERN SPL beam and beta beams is described in the CERN yellow report M. Apollonio et al., hep-ph/0210192

30. There are many articles discussing neutrino factory capabilities, see for example J.J. Gomez-Cadenas and D.A. Harris, Ann. Rev. Nucl. and Part. Sci. 52 253, (2002) and references therein.

31. See for example, P. Huber and W. Winter, Phys.Rev. D68, 037301,2003, A. Asratyan et al, hep-ex/0303023

32. MUCOOL R\& D web page: http://www.fnal.gov/projects/ muon_collider/cool/cool.html, and papers submitted to Accelerator R\& D session at EPS 2003: http://eps2003.physik.rwth-aachen.de/

33. M. Komatsu, P. Migliozzi, F. Terranova, J.Phys.G29 443, 2003

34. M.Diwan, M.Messier, B.Viren, L.Wai, NUMI-L-714

35. A. Rubbia, P. Sala, JHEP 209 4,2002

36. Mauro Mezzetto, private communication

37. "Parameters of Radiological Interest for a Betabeam decay ring" M. Magistris and M. Silari, Technical Note in preparation 


\section{DISCUSSION}

Antonio Ereditato (INFN-Napoli): A remark about your concerns on the LAr TPC technique: a 600-ton ICARUS module has been successfully tested at the surface with cosmic rays. At the same Web site from which you picked up MC events, you may find a "real event" gallery and a list of published papers using these events.

Deborah Harris: I agree that much progress has been made recently with event reconstruction. It will still be very important however to see how well this detector does in discriminating between $\pi^{0}$ 's and single electrons. I would certainly be very happy if this technology were the one that gets used in the long term future!

Peter Rosen (DOE): Have you thought much about the measurement of neutral currents? One of the beautiful features of solar neutrinos is SNO's ability to do that.

Deborah Harris: The superbeam experiments described here have really been optimized for $\nu_{\mu}$ to $\nu_{e}$ searches. At the limit they have considered how well the $\nu_{\mu}$ disappearance probability could be measured. However, as far as I know the only analysis done on using neutral current events, either to search for $\nu_{\tau}$ "appearance" or to search for the sterile neutrino sector, is the MINOS experiment.

Joe Formaggio (Univ. of Washington): Doesn't the atmospheric neutrino background [for a $\beta$ beam experiment] get reduced by beam timing?

Deborah Harris: If you were nominally trying to build a $\beta$-beam, you would simply fill the decay ring with relativistic ions, and then you would have basically no timing. However, by working out how to impose a bunch structure in a $\beta$-beam you not only reduce the atmospheric background, but you also allow the possibility of putting $\mathrm{He}$ and $\mathrm{Ne}$ (and thereby making $\nu_{e}$ and $\bar{\nu}_{e}$ ) in different bunches.

Bennie Ward (Univ. of Tennessee): In the study of $\mathrm{CP}$ violation in the quark sector, we're used to discovery in terms of many, many sigma. Could you comment on the use of a 3 sigma discovery criterion in the lepton sector?
Deborah Harris: I think it's a measre of how difficult these experiments are to do in the first place. With superbeams and what we know of today's technology, there is only a small region of parameter space where even maximal CP violation can be seen, because of the inherent backgrounds and how well one can reasonably expect to ever know them. In contrast, neutrino factory beams provide measurements with two orders of magnitude lower backgrounds and high rates, and is certainly the only place we know of now where $\mathrm{CP}$ violating effects could possibly be seen at "many many sigma".

Ed Witten (Princeton University): I would think that the radiation levels at a $\beta$-beam facility would be prohibitively large-is this something that has been addressed?

Deborah Harris: This is, as you suspect, a serious concern to people designing $\beta$-beams. The extimated radioactivity level for a facility with the fluxes described in this report is $8.5 \mathrm{Watt} / \mathrm{m}$, while $1 \mathrm{Watt} / \mathrm{m}$ is the standard security limit for an accelerator ${ }^{36}$. What is even more worrisome is that this radioactivity tends to accumulate at the end of the straight sections. Although special care needs to be taken for the proper disposal of the materials in the ring after the experiment is over, with proper design the doses to the magnets themselves can be reduced to an acceptable level. See reference ${ }^{37}$ for a recent calculation of the dose levels. 